\title{
Second harmonic surface response of a composite
}

\author{
Bernardo S. Mendoza ${ }^{a}$ and W. Luis Mochán ${ }^{b}$ \\ ${ }^{a}$ Department of Photonics, Centro de Investigaciones en Optica, León, Guanajuato, México; \\ ${ }^{b}$ Laboratorio de Cuernavaca, Instituto de Física, Universidad Nacional Autónoma de México, \\ Apartado Postal 48-3, 62251 Cuernavaca, Morelos, México.
}

\begin{abstract}
We calculate the nonlinear dipole and quadrupole moments induced at the second harmonic (SH) frequency $2 \omega$ in a dielectric nanosphere by an inhomogeneous monochromatic electric field of frequency $\omega$. We neglect finite size effects and assume that the selvedge region of the nanosphere is thin enough so that the surface may be considered locally flat. Then, we calculate the nonlinear optical response of a centrosymmetric semiinfinite composite made by the nanospheres. Within the dipole approximation, $\mathrm{SH}$ is forbidden in the centrosymmetric bulk, but allowed at the surface of the composite where the symmetry is broken. Therefore, the components of the surface susceptibility tensor, $\chi_{i j k}$, different from zero are calculated. As an application, we evaluate $\chi_{i j k}$ for a composite made of $\mathrm{Si}$ nanospheres.
\end{abstract}

Keywords: Second harmonic generation, nanoparticles, composites

\section{INTRODUCTION}

The use of nonlinear optical techniques to study surface phenomena has gained a lot of attention in recent years. In particular second harmonic generation (SHG) has been established as a very powerful spectroscopy to study a wide range of physical and chemical phenomena at the surface centrosymmetric materials. ${ }^{1-3}$ The surface sensitivity of SHG is due to the fact that within the dipole approximation a centrosymmetric environment does not radiate $\mathrm{SH}$, while the inversion symmetry is broken at its surface, thus allowing the radiation of SH. On the experimental side, the new tunable high intensity laser systems have made SHG spectroscopy readily accessible and applicable to a wide range of systems. ${ }^{2,3}$ However, the theoretical development of the field is still an ongoing subject of research. Some recent advances for the case of semiconducting and metallic systems have appeared in the literature, where the confrontation of theoretical models with experiment has succeeded, yielding correct physical interpretations for the SHG spectra. ${ }^{4-9}$ Most of the SHG studies, both theoretical and experimental, have been concerned with planar surfaces, and much less attention has been paid to nonplanar systems. Novel nanofabrication techniques are now capable of producing nanoparticles with controlled structures which include small clusters, self-assembled particles, quantum dots, vesicles, etc. The nonlinear optical properties of these structures are important for applications, and can be used for their physical characterization. ${ }^{10,11}$

There have been few theoretical investigations dealing with the SH behavior of small spheres. Most of them make use of very restrictive models for the intrinsic bulk ${ }^{12,13}$ or surface ${ }^{14,15}$ nonlinear optical response of the sphere. The centrosymmetry of a nanoparticle is locally disrupted by its surface. However, it is regained globally for a spherical shape. Thus, a homogeneous polarizing field induces mutually canceling polarizations at opposite sides of the sphere, and would be incapable of producing dipolar radiation; it would produce only higher multipolar radiation with a power of leading order $k^{6}$, where $k$ is the wavenumber. However, for a nonuniform polarizing field, this cancellation is no longer exact and dipolar radiation becomes possible. Recently Dadap et $a l .{ }^{16,17}$ presented a model that permits an arbitrary nonlinear intrinsic response, and calculated the SH Rayleigh scattering from a small sphere of centrosymmetric material illuminated by a linearly or circularly polarized plane wave. Retardation of the incoming field across the particle leads to a total dipole moment that scales as $R / \lambda$ for small spheres, where $R$ is the radius of the sphere and $\lambda$ the wavelength. Thus, although retardation leads to dipolar radiation, it is of order $k^{6}$, and therefore comparable to the quadrupolar radiation, instead of the usual linear dipolar Mie scattering which is of order $k^{4}$ and dominates over the quadrupolar scattering.

Further author information: bms@cio.mx 
The field variations of a plane wave due to retardation take place necessarily along its wavevector, which is perpendicular to the polarizing field, and with concomitant lengthscale $\lambda$. However, in many systems of interest, such as in the neighborhood of other particles or of a substrate, the field may have variations with other lengthscales and along different directions.

Since for small lengthscales the polarizing field is mostly longitudinal, our purpose in this paper is the calculation of the SHG by a centrosymmetric semiinfinite composite of small spherical particles subject to an inhomogeneous longitudinal field. To this end, we extend the model developed originally to obtain SHG for a single dielectric sphere. ${ }^{19}$ This model allowed a closed analytical solution for the surface and bulk nonlinear susceptibilities in terms of the linear dielectric function of the system. Here too, we consider a particle of radius $R \ll \lambda$ but large enough so that we may assume those results to be locally valid inside and at the surface of the sphere. Thus, we obtain simple analytical expressions for the dipolar nonlinear susceptibilities of the composite surface. Their use permits a detailed analysis of the spectral features of the SHG radiated power. As an application, we calculate the susceptibility of a composite made of Si nanospheres.

The structure of the paper is the following: In Sec. 2 we show the theory of the response of a sphere placed above a semiinfinite inert dielectric substrate with a flat surface, interacting at the fundamental and $\mathrm{SH}$ frequencies with its own dipole and quadrupole image fields. Then, we obtain the nonlinear susceptibility of a composite of such spheres, and present the results and conclusions in Sec. 3, for a composite of Si nanospheres.

\section{THEORY}

The system we want to study consists of a continuous distribution of nanospheres of radius $R$, that extends from the surface of a semiinfinite system to its centrosymmetric bulk at $z \rightarrow \infty$ (see Fig. 1). Each sphere interacts only with its image created by the presence of the surface. First we write the total polarization of each sphere in the presence of its image, and then we integrate this polarization over $z$ to obtain the total response of the system. Following Ref. 19, we write the main results that we need in order to calculate the response of the semiinfinite composite. For a single sphere (in the presence of its image), the total polarization, $p_{i}$, induced by the external electromagnetic field, $\mathbf{E}$, is given by

$$
p_{z}=p_{z}^{n l}-\beta_{2}^{d} \frac{1-\epsilon_{2}^{s}}{1+\epsilon_{2}^{s}}\left(\frac{p_{z}}{4 z^{3}}+\frac{3 Q_{y y}^{n l}}{64 z^{4}}\right),
$$

for the case in which $\mathbf{E}$ is $s$ polarized. On the other hand, for $p$ polarized incoming light,

$$
\begin{aligned}
& p_{x}=p_{x}^{n l}-\beta_{2}^{d} \frac{1-\epsilon_{2}^{s}}{1+\epsilon_{2}^{s}}\left(\frac{p_{x}}{8 z^{3}}-\frac{Q_{x z}^{n l}}{16 z^{4}}\right), \\
& p_{z}=p_{z}^{n l}-\beta_{2}^{d} \frac{1-\epsilon_{2}^{s}}{1+\epsilon_{2}^{s}}\left(\frac{p_{z}}{4 z^{3}}-\frac{3 Q_{z z}^{n l}}{32 z^{4}}\right) .
\end{aligned}
$$

In above equations, the nonlinear dipole, $p_{i}^{n l}$, and quadrupole, $Q_{i j}^{n l}$, induced on the sphere by the inhomogeneous local field may be written as

$$
p_{i}^{n l}=\chi_{i j k}^{d} E_{j} E_{k}
$$

and

$$
Q_{i j}^{n l}=\chi_{i j k l}^{Q} E_{k} E_{l}
$$

where

$$
\chi_{i j k}^{d}=\gamma^{d} \eta_{l j} \eta_{l i k}
$$

and

$$
\chi_{i j k l}^{Q}=\frac{1}{2} \gamma^{Q}\left(\eta_{i k} \eta_{j l}+\eta_{i l} \eta_{j k}-\frac{2}{3} \delta_{i j} \eta_{m k} \eta_{m l}\right)
$$

represent the nonlinear response of the sphere to the macroscopic field, $E_{i}$. In all equations repeated indices are summed. Also,

$$
\eta_{i j}=\left(\delta_{i j}-T_{i j}^{I} \beta_{1}^{d}\right)^{-1}
$$




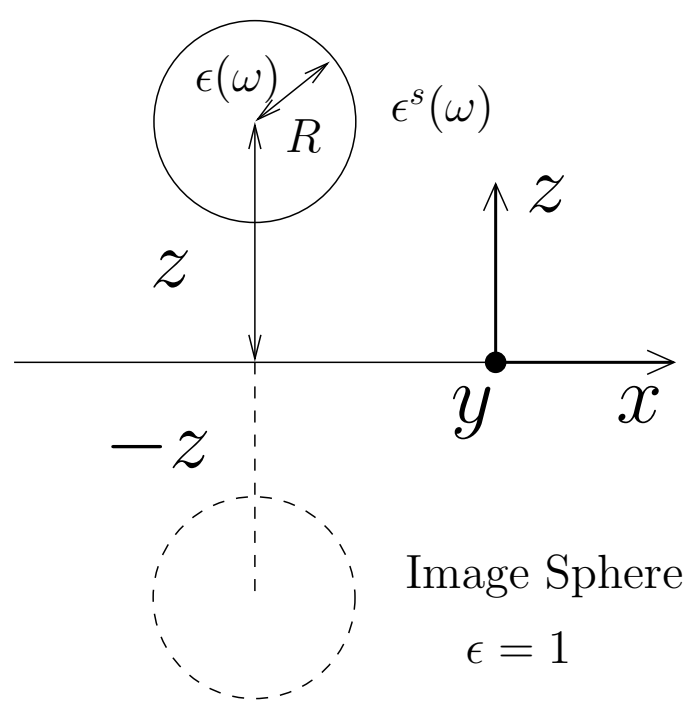

Figure 1. The semiinfinite system consist of a continuous distribution of noninteracting spheres of radius $R$ with dielectric function $\epsilon(\omega)$ embedded in a substrate with dielectric function $\epsilon^{s}(\omega)$. The image sphere contains the image dipole and quadrupole of the original sphere located at $x=y=0$ and $z$. The origin of the coordinate system is shifted to the right for clarity.

is the 'quotient' between the local and the macroscopic fields, $E_{i}^{l o c}=\eta_{i j} E_{j}$, and

$$
\eta_{i j k} \equiv \partial_{i} \eta_{j k}=\eta_{j l} T_{i l m}^{I} \beta_{1}^{d} \eta_{m k},
$$

with

$$
T_{i j k}^{I} \equiv \partial_{i} T_{j k}^{I}=T_{i j l} S_{l k} \frac{1-\epsilon_{1}^{s}}{1+\epsilon_{1}^{s}}
$$

and

$$
T_{i j k}=\partial_{i} T_{j k}=\partial_{i} \partial_{j} \partial_{k} \frac{1}{\left|\vec{r}^{d}-\vec{r}^{I}\right|} .
$$

$T_{i j}^{I}$ is the image mediated dipolar self-interaction tensor, and $T_{i j k}^{I}$ is also the image mediated self-interaction tensor, but for the nonlinear response. We have taken $\mathbf{r}^{d}=(0,0, z)$ and $\mathbf{r}^{d}-\mathbf{r}^{I}=(0,0,2 z)$, and abbreviate the gradients $\partial_{i} \equiv \partial / \partial r_{i}^{d}$ acting on $\vec{r}^{d}$ while keeping the position of the image $\vec{r}^{I}$ constant. $S=\operatorname{diag}(1,1,-1)$ is the $z \rightarrow-z$ reflection operator. Here we introduce the dielectric function $\epsilon_{n}^{s} \equiv \epsilon^{s}(n \omega)$ of the substrate, that of the sphere $\epsilon_{n} \equiv \epsilon(n \omega)$, with $n=1,2$, and the linear dipolar polarizability $\beta_{m}^{d}=\beta^{d}(m \omega)$ is given by ${ }^{20}$

$$
\beta^{d}(\omega)=\frac{\epsilon(\omega)-1}{\epsilon(\omega)+2} R^{3} .
$$

Finally, the dipolar and quadrupolar nonlinear polarizabilities of the isolated sphere are given by

$$
\gamma^{d}=\frac{1}{8 \pi n e} \frac{\epsilon_{1}-1}{\left(\epsilon_{1}+2\right)\left(2 \epsilon_{1}+3\right)}\left(15 \frac{\epsilon_{1}-2 \epsilon_{2}+1}{\epsilon_{2}+2}+\left(\epsilon_{1}-1\right)(2 a+b+3 f)\right) R^{3},
$$

and

$$
\gamma^{Q}=\frac{9}{20 \pi n e} \frac{\left(\epsilon_{1}-1\right)^{2}}{\left(\epsilon_{1}+2\right)^{2}}(a+3 b-f) R^{3},
$$

where $n$ is the number density of the polarizable entities that make up a single sphere, $-e$ is the charge of an electron, and $a=a(\omega), b=b(\omega)$ and $f=f(\omega)$ are dimensionless functions which are commonly employed to 
parametrize the response of the surface. ${ }^{19}$ One can identify bulk and surface contributions to $\gamma^{d}$, the latter being proportional to the surface parameters $a, b$ and $f$. It is interesting to note that both contributions scale with the volume of the sphere and not with its area. The surface contribution to $\gamma^{d}$ inherits the resonant structure of $a$, $b$ and $f$, while its bulk contribution has resonances at frequencies for which the second harmonic depolarization field excites a dipolar plasmon, given by the condition $\epsilon_{2}=-2$. There are further resonances in both terms corresponding to the excitation of dipolar and quadrupolar plasmons at the fundamental frequency, given by the conditions $\epsilon_{1}=-2$ and $\epsilon_{1}=-3 / 2$ respectively. The quadrupolar response (Eq. (14) also scales with the volume of the sphere. It may seem paradoxical that it contains only a 'surface' term, since each 'molecule' within the sphere has a finite quadrupole moment. The reason is that $a, b$ and $f$ necessarily contain bulk contributions besides intrinsic surface contributions, ${ }^{21}$ so that the bulk quadrupolar contribution is actually present but hidden within the 'surface contribution'. Besides the resonances of the surface parameters, $\gamma^{Q}$ has only a dipolar (double) resonance at the fundamental frequency.

Now, we can solve Eqs. (1), (2) and (3) for the polarization, p, that as can be seen from these equations and Eq. (11) depends on the position of the sphere, $z$, away from the surface. Therefore the total response of the system is given by

$$
p_{i}^{t o t}=\int_{0}^{\infty} p_{i}(z) d z
$$

which can be written as

$$
p_{i}^{t o t}=\chi_{i j k} E_{j} E_{k},
$$

with $\chi_{i j k}$ the nonlinear surface susceptibility of the system. The integration of Eq. (15) is straightforward, and after some algebra we obtain the following nonzero components of $\chi_{i j k}$,

$$
\begin{gathered}
\chi_{z z z}=\gamma^{Q}\left[\frac{\eta^{2} \log \left(\frac{1+f_{2} / 4}{1+f_{1} / 4}\right)}{12(\eta-1)^{2}}-\frac{\eta}{12(\eta-1)\left(1+4 / f_{1}\right)}\right] \\
+\gamma^{d}\left[\frac{1}{4(\eta-1)\left(1+4 / f_{1}\right)^{2}}-\frac{2 \eta-1}{2(\eta-1)^{2}\left(1+4 / f_{1}\right)}-\frac{\eta^{2} \log \left(\frac{1+f_{2} / 4}{1+f_{1} / 4}\right)}{2(\eta-1)^{3}}\right], \\
\chi_{x x z}=-\frac{1}{3} \gamma^{Q}\left[\frac{\log \left(\frac{1+f_{1} / 4}{1+f_{2} / 8}\right)}{1-2 / \eta}+\frac{\log \left(\frac{1+f_{2} / 8}{1+f_{1} / 8}\right)}{2(1-1 / \eta)}\right],
\end{gathered}
$$

and

$$
\begin{aligned}
\chi_{x x z} & =\gamma^{Q}\left[\frac{\eta}{12(2 \eta-1)\left(1+8 / f_{1}\right)}+\frac{\eta^{2} \log \left(\frac{1+f_{1} / 8}{1+f_{2} / 4}\right)}{6(2 \eta-1)^{2}}\right] \\
& +\gamma^{d}\left[\frac{1}{2(2 \eta-1)\left(1+8 / f_{1}\right)}-\frac{(3 \eta-1) \log (2)}{(2 \eta-1)^{2}}+\frac{\eta^{2} \log \left(4+f_{2}\right)}{(\eta-1)(2 \eta-1)^{2}}-\frac{\log \left(4+f_{1}\right)}{\eta-1}+\frac{(3 \eta-1) \log \left(8+f_{1}\right)}{(2 \eta-1)^{2}}\right],
\end{aligned}
$$

where $f_{n}=\beta_{n} g_{n}$,

$$
\begin{aligned}
& \beta_{n}=\frac{\epsilon(n \omega)-1}{\epsilon(n \omega)+2}, \\
& g_{n}=\frac{1-\epsilon(n \omega)}{1+\epsilon(n \omega)},
\end{aligned}
$$

with $n=1,2$, and $\eta=f_{2} / f_{1}$. Here, $\epsilon(\omega)$ is the dielectric function of the sphere. From symmetry, $\chi_{z y y}=\chi_{z x x}$, $\chi_{x x z}=\chi_{y y z}$, and $\chi_{i j k}=\chi_{i k j}$. Eqs. (17), (18), and (19), are the main result of the article. It is worth mentioning that the nonlinear susceptibility only depends on the macroscopic dielectric function of the sphere, $\epsilon(\omega)$, the substrate, $\epsilon^{s}(\omega)$ and the sphere surface parameters $a(\omega), b(\omega)$ and $f(\omega)$. 


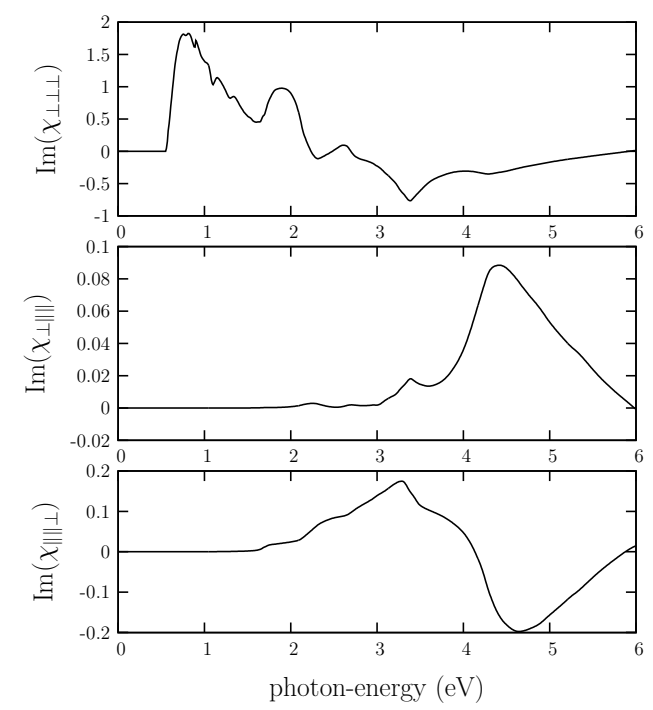

Figure 2. Plot of the $\operatorname{Im}\left(\chi_{i j k}\right)$ vs. the incident photon energy.

\section{RESULTS AND CONCLUSIONS}

In this section we evaluate $\chi_{i j k}$ of Eqs. (17), (18), and 19. To do so, we use the continuous dipolium model for a flat homogeneous surface of Ref. 18, from which the explicit expression for the $a(\omega)$ parameter is given by,

$$
a=2\left(\left[\epsilon_{2}-\epsilon_{1}\right]\left[2 \epsilon_{1}-\epsilon_{2}-\epsilon_{1} \epsilon_{2}\right]+\left[\epsilon_{1}\right]^{2}\left[1-\epsilon_{2}\right] \log \left[\epsilon_{1} / \epsilon_{2}\right]\right) /\left[\epsilon_{2}-\epsilon_{1}\right]^{2},
$$

as well as the results $b=-1, f=0$. With Eq. (22) is now possible to evaluate $\chi_{i j k}$ with only knowing the dielectric function of the sphere, $\epsilon$, at the fundamental frequency $\omega$ and the second harmonic frequency $2 \omega$.

As an example we use $\mathrm{Si}$, since recently, composites of $\mathrm{Si}$ nanospheres have been successfully investigated with SH. ${ }^{10}$ Therefore, we use the dielectric function of Si taken from Ref. 22. In Fig. 2 we show the imaginary part of $\chi_{i j k}$, where we have used $\|$ for the parallel direction to the surface $(x$ or $y)$, and $\perp$ for the $z$ direction. Therefore, the only components different from zero are $\chi_{\perp \perp \perp}, \chi_{\perp\|\| \|}$, and $\chi_{\|\| \perp}=\chi_{\|\perp\|}$. Also, we have rescaled $\chi_{i j k}$ to include the $R^{3} /(e n)$ factor that comes from $\gamma^{d}$ and $\gamma^{Q}$ of Eqs. (13) and (14), respectively. As we see, $\chi_{\perp \perp \perp}$ is the dominant component for the energies shown, that cover all the optical spectrum. At energies between 3 and $5 \mathrm{eV}, \chi_{\perp\|\|}$ and $\chi_{\|\| \perp}$ are similar in size to $\chi_{\perp \perp \perp}$. The different 'peaks' and 'valleys' in the spectra are related to the resonances of $a(\omega)$, which in turn can be related back to the $\mathrm{E}_{1}$ and $\mathrm{E}_{2}$ resonances of the bulk critical points of $\mathrm{Si}$, their subharmonics, and those of the dipolar and quadrupolar resonances of the isolated sphere. The real part of $\chi_{i j k}$ shows similar behavior to that of the imaginary part. It is important to note that, $\chi_{\perp\|\| \|}$ and $\chi_{\|\| \| \perp}$ can be related to a $b(\omega)$ and $f(\omega)$, respectively, that now will be giving the response of the composite surface. As we see from Fig. 2, the composite's $f(\omega) \neq 0$, and $b(\omega)$ has a dispersion different from a simple constant, thus giving a different result from what one obtains for a flat surface jellium, or in this case, for the isolated sphere, for which $b=-1$ and $f=0$.

With the nonlinear susceptibility $\chi_{i j k}$, one can proceed to calculate the radiated nonlinear SH efficiency, where formalisms like the 'three layer model' of Ref. 23 can be used. Then, one can compare with spectroscopic measurements for this kind of systems, for which the experiments of Ref. 10, could serve as an starting point.

In conclusion, we have presented a derivation for the second order nonlinear susceptibility of a noninteracting composite of nanospheres. The nonzero components of $\chi_{i j k}$ are analytic functions of the dielectric function of the host, that of the nanosphere material, and the parameters that describe the 'surface' SH response of the nanosphere. As an example we have calculated $\chi_{\perp \perp \perp}, \chi_{\perp\|\| \|}$, and $\chi_{\|\| \perp}$, for Si nanospheres, and shown their rich spectroscopic behavior. Our formalism, represent an starting point to study, through nonlinear optics, this interesting nonoscale systems. 


\section{ACKNOWLEDGMENTS}

This work was supported by CONACyT 36033-E (Mendoza) and Grant No. DGAPA-UNAM-IN117402 (Mochán).

\section{REFERENCES}

1. For a recent review see, 5th International Conference on Optics of Surfaces and Interfaces, Edited by B. Mendoza, physica status solidi (c) 0 Num. 8 (2003).

2. M.C. Downer, B.S. Mendoza and V.I. Gavrilenko, Surf. Interface Anal. 31, 966-986 (2001).

3. G. Lüpke, Surf. Sci. Reports 35, 75 (1999).

4. P. Guyot-Sionnest, A. Tadjedinne, and A. Liebsch, Phys. Rev. Lett. 64, 1678 (1990).

5. B. S. Mendoza and W. L. Mochán, Phys. Rev. B 53, 10473 (1996).

6. B. S. Mendoza and W. L. Mochán, Phys. Rev. B 55, 2489 (1997).

7. B. S. Mendoza, A. Gaggiotti, and R. D. Sole, Phys. Rev. Lett. 81, 3781 (1998).

8. B. S. Mendoza, W. L. Mochán, and J. A. Maytorena, Phys. Rev. B 60, 14334 (1999).

9. D. Lim, M. C. Downer, J. G. Ekerdt, N. Arzate, B. S. M. V. Garvrilenko, and R. Wu, Phys. Rev. Lett. 84, $3406(2000)$.

10. P. Figliozzi, L. Sun, Y. Jiang, N. Matlis, B. Mattern, M.C. Downer, S.P. Withrow, C.W. White, W.L. Mochán, and B.S. Mendoza, Phys. Rev. Lett. 94, 047401 (2005).

11. L. Sun, P. Figliozzi, Y.Q. An, M.C. Downer, W.L. Mochán, and B.S. Mendoza, Optics Letters, in press.

12. G. S. Agarwall and S. S. Jha, Solid State Commun. 41, 499 (1982).

13. X. M. Hua and J. I. Gersten, Phys. Rev. B 33, 3756 (1986).

14. D. Östling, P. Stampfli, and K. H. Bennemann, Z. Phys. D 28, 169 (1993).

15. J. Martorell, R. Vilaseca, and R. Corbalán, Phys. Rev. A 55, 4520 (1997).

16. J. I. Dadap, J. Shan, K. B. Eisenthal, and T. F. Heinz, Phys. Rev. Lett. 83, 4045 (1999).

17. J. I. Dadap, J. Shan, K. B. Eisenthal, and T. F. Heinz, J. Opt. Soc. Am. B 21, 1328 (2004).

18. B. S. Mendoza and W. L. Mochán, Phys. Rev. B 53, 4999 (1996). Erratum: Phys. Rev. B 61, 16243 (2000).

19. Vera L. Brudny, Bernardo S. Mendoza and W. Luis Mochán, Phys. Rev. B 62, 11152-11162 (2000).

20. J. D. Jackson, Classical Electrodynamics (John Wiley and Sons, NY, 1975), second ed.

21. A. Petukhov, V. L. Brudny, W. L. Mochán, J. Maytorena, B. S. Mendoza, and Th. Rasing, Phys. Rev. Lett. 81, 566 (1998).

22. D.F. Edwards, Handbook of Optical Constants of Solids, edited by E. Palik, (Academic, New York, 1985), p. 552.

23. J. Mejía, Bernardo S. Mendoza, M. Palummo, G. Onida, R. Del Sole, S. Bergfel and W. Daum, Phys. Rev. B 66, 195329 (2002). 